\title{
ABHD6 inhibition rescues a sex-dependent deficit in motor coordination in the HdhQ200/200 mouse model of Huntington's disease
}

\author{
Jessica K. Cao \\ University of Washington \\ Katie Viray \\ University of Washington \\ Myungsun Shin \\ University of Virginia \\ Ku-Lung Hsu \\ University of Virginia \\ Ken Mackie \\ Indiana University Bloomington \\ Ruth Westenbroek \\ University of Washington \\ Nephi Stella ( $\nabla$ nstella@uw.edu ) \\ University of Washington
}

\section{Research Article}

Keywords: Huntington's Disease, ABHD6 inhibition

Posted Date: February 24th, 2021

DOI: https://doi.org/10.21203/rs.3.rs-230223/v1

License: (9) This work is licensed under a Creative Commons Attribution 4.0 International License.

Read Full License 


\section{ABHD6 inhibition rescues a sex-dependent deficit in motor coordination in the HdhQ200/200 mouse model of Huntington's disease}

${ }^{1}$ Department of Pharmacology, University of Washington School of Medicine, Seattle, WA 98195, USA.

${ }^{2}$ Department of Chemistry, University of Virginia, Charlottesville, VA 22904, USA.

${ }^{3}$ Department of Psychological and Brain Sciences, Linda and Jack Gill Center for Biomolecular Science, Indiana University, Bloomington, IN 47405, USA.

${ }^{4}$ Department of Psychiatry and Behavioral Sciences, University of Washington School of Medicine, Seattle, WA 98195, USA.

*Author for Correspondence:

Dr. Nephi Stella

Address: HSC BB1504A

1959 NE Pacific St

Seattle, WA 98195-7280

Phone: 206-221-5220

Fax: 206-543-9520

E-mail: nstella@uw.edu

Running title: ABHD6 and Huntington's disease

Number of Figures: 5

Abbreviations:, 2-arachidonoyl glycerol (2-AG); alpha/beta-hydrolase domain containing 6 (ABHD6); activity-based protein profiling (ABPP), cannabinoid receptor $1\left(\mathbf{C B}_{1} \mathbf{R}\right)$; diacylglycerol lipase (DAGL), endocannabinoids (eCB); fatty acid amide hydrolase (FAAH); gamma-aminobutyric acid (GABA); Huntington's disease (HD); immunohistochemistry (IHC); monoacylglycerol lipase (MAGL); mutated huntingtin protein (mtHTT); medium spiny neurons (MSN); semi-quantitative immunohistochemistry (sqIHC) and wildtype (WT). 
Abstract (188 words)

34 Huntington's Disease is associated with motor behavior deficits that current therapeutics do not alleviate. 35 This pilot study tested if pharmacological inhibition of $\alpha / \beta$-hydrolase domain containing 6 (ABHD6), a multifunctional enzyme expressed in the striatum, rescues behavioral deficits in HdhQ200/200 mice. Previous work has shown that this model exhibits a reduction in spontaneous locomotion and motor coordination at 8 and 10 months of age, with a more severe phenotype in female mice. Semi-quantitative immunohistochemistry analysis indicated no change in striatal ABHD6 expression at 8 months of age, but a 40\% reduction by 10 months in female HdhQ200/200 mice compared to female wild-type (WT) littermates. At 8 months of age, acute ABHD6 inhibition selectively rescued motor coordination deficits in female HdhQ200/200 mice without affecting W'T performance. ABHD6 inhibition did not impact spontaneous locomotion, grip strength or overall weight in either group, showing that effects were specific to motor coordination. At 10 months of age, semi-chronic ABHD6 inhibition by osmotic pump delivery also rescued motor coordination deficits in female HdhQ200/200 mice without affecting female W'T littermates. Our preliminary study suggests that ABHD6 inhibition selectively improves motor performance in female HdhQ200/200 mice.

\section{Introduction}

Huntington's Disease (HD) is characterized by age-dependent deterioration of locomotor and cognitive huntingtin proteins $(\mathbf{m t H T} \mathbf{T})$ produced by excessive CAG repeats in the HTT gene. These mutated proteins

52 interfere with normal neuronal functions through a multitude of mechanisms ${ }^{1,2}$. HD pathological studies showed that mtHTT expression leads to early dysfunction of select genes and proteins in striatal neurons ${ }^{3,4}$. Considering that few FDA-approved therapeutics are effective for the treatment of HD and most carry the risk of severe side effects, there is an urgent need to better understand the molecular components affected in HD pathogenesis to help develop new therapeutic approaches for safer treatment.

The genetic basis of HD has led to the development of preclinical mouse models that recapitulate molecular mechanisms involved in HD pathogenesis. The HdhQ mouse model was developed using gene targeting technology to insert CAG repeats in the Hdh gene, the mouse homolog to HTT ${ }^{5}$. Natural germline increases in the expansion of CAG in $\mathrm{Hdh}$ of the HdhQ150/+ mouse line was leveraged to create several

61 allelic mouse lines, including the HdhQ200/+ and HdhQ200/200 lines ${ }^{6,7}$. Studies showed that the initial

62 aspects of HD neuropathology and impaired locomotor behavior were detected in the heterozygous 63 HdhQ200/+ line starting at 12 months of age and reached severe impairment by 18 months of age; however, $64 \mathrm{HdhQ} 200 /+$ mice maintained a normal life span of approximately 2 years, failing to mimic the reduced life 
span characteristic of human adult-onset $\mathrm{HD}^{8}$. Our laboratory reported the longitudinal characterization of the behavioral impairment and neuropathological features modeled by the homozygous HdhQ200/200 line . HD pathogenesis was sex-dependent, exhibited an early onset of symptoms through deficits in motor behavior and impaired striatal medium spiny neuron (MSN) function at 8 months of age, and was fatal, resulting in a reduced lifespan of 12 months in this model ${ }^{7}$. Female HdhQ200/200 mice displayed a more robust deterioration in spontaneous locomotion and motor coordination, a result that corresponds with human studies showing that women HD patients present with more severe symptoms regardless of CAG expansion $\operatorname{size}^{9,10}$. A more recent study uncovered that there was a significantly higher prevalence of HD in women than men, suggesting a more severe HD pathological process in women ${ }^{11}$. Thus, the early and more severe onset of impaired motor behavior detected in female HdhQ200/200 mice provides an opportunistic chance to study the involvement of select molecular mechanisms in HD-associated deficits in locomotor behaviors and explore possible avenues for new therapeutics.

The recently discovered multifunctional enzyme $\alpha / \beta$-hydrolase domain containing 6 (ABHD6) regulates several facets of neuronal functions, including inhibiting neurotransmitter release through the modulation of endocannabinoid (eCB) signaling, increasing the amplitude of postsynaptic GABAergic currents through allosteric modulation, and controlling the trafficking and number of post-synaptic AMPA receptors ${ }^{12}$. This suggests that changes in ABHD6 expression and activity are likely to regulate neuronal functions in multiple ways. mRNA expression profiling suggests that ABHD6 is particularly abundant in the striatum ${ }^{13}$. We previously developed a rabbit polyclonal antibody that recognizes mouse ABHD6 protein and provided initial immunohistochemistry (IHC) evidence that ABHD6 protein is expressed in the striatum ${ }^{14}$, however it is unknown how various diseases affect this expression. Recent studies suggest that ABHD6 inhibitors exhibit promising therapeutic efficacy in several preclinical mouse models of neurological diseases, including seizures, traumatic brain injury, and multiple sclerosis ${ }^{15-18}$. Importantly, the therapeutic efficacy of ABHD6 inhibitors does not appear to undergo tolerance or trigger overt side-effects, two positive qualities for potential smallmolecule therapeutics ${ }^{18}$. Therefore, we conducted a pilot study to examine if levels of ABHD6 were affected in HD and tested whether ABHD6 inhibition, either through acute or semi-chronic delivery, can rescue various behavioral deficits in the HdhQ200/200 mouse model.

\section{Materials and Methods}

\subsection{HdhQ200/200 mouse colony.}

Mice were housed in a pathogenic-free facility in accordance with the National Institutes of Health; the Institutional Animal Care and Use Committee at the University of Washington approved all experiments and in compliance with ARRIVE guidelines. All mice were maintained on a C57BL/6 genetic background. For all HdhQ200/200 experiments, female and male heterozygous HdhQ200/+ knock-in mice were mated to 
produce homozygous, heterozygous and wild-type littermates. Animals were housed in cages grouped by sex

99

100

101

102

103

104

105

106

107

108

109

110

111

112

113

114

115

116

117

118

119

120

121

122

123

124

125

126

127

128

129

130 and mixed genotype, had ad libitum access to food and water, and were on a 12-hour light/dark cycle. Genotyping was performed with tail snips using primers "cccattcattgccettgctg" and "gcggctgagggggttga" with a SimpliAmp Thermal Cycler (Thermo Fisher Scientific, Bothell, WA). No sexual dimorphism was detected in the HdhQ200/200 colony (i.e. no differences in litter size or weight ${ }^{7}$. Mice lacking Abhd6 were generated in collaboration with the TSRI Mouse Genetics Core and the absence of ABHD6 expression validated ${ }^{19}$. A total number of animals were used for this study.

\subsection{Immunohistochemistry and antibodies.}

Mice were euthanized with ketamine/xylazine and perfused with PBS, followed by 4\% paraformaldehyde. Brains were extracted and post-fixed in $4 \%$ paraformaldehyde at $4^{\circ} \mathrm{C}$. Brains were then dehydrated $(15 \%$ sucrose and then 30\% sucrose) and frozen over dry ice. Sagittal sections were cut to a thickness of $30 \mu \mathrm{m}$ using a sliding microtome and placed in cryoprotectant for storage at $-20^{\circ} \mathrm{C}$. IHC analysis of each time point group was processed and stained in parallel. For staining, 2 slices per animal were removed from cryoprotectant, washed and incubated at room temperature in blocking buffer. Slices were then transferred to primary staining solutions at $4^{\circ} \mathrm{C}$. Sections were then washed and secondary staining was performed using Alexa secondary antibodies at a dilution of 1:500. Additional slices were stained only with secondary staining solutions to serve as non-specific secondary background staining. After secondary staining, sections were washed, mounted on slides and allowed to dry at room temperature overnight. Coverslips were added and sealed with Fluoromount (Sigma, St. Louis, MO) and nail polish. The following antibodies were used in this study: ABHD6 ${ }^{14}$ and Alexa Fluor 488 (goat/donkey, ThermoFisher).

\subsection{Microscopy.}

Images were collected on a Marianas microscope (Intelligent Imaging Innovations, Inc. Denver, CO) equipped with either 20x/0.75 NA or 40x/0.75 NA air objective lens with CoolSnap HQ cooled monochrome camera (Photometrics, Tucson, AZ) at the NIDCD Research Core Center at the University of Washington, as previously reported by our laboratory ${ }^{20,21}$. Noise reduction of images was achieved by deconvolution using SlideBook (Intelligent Imaging Innovations, Inc., Denver, CO). Exposure times were optimized for each antibody to ensure that $>99 \%$ pixels were within the linear range. Excitation laser of FITC was used to excite excite Alexa 488.

\subsection{Semi-quantitative image analysis and statistics.}

All images were analyzed using ImageJ (National Institutes of Health) with custom written macros. Macros were applied blindly to each batch of images and analyzed as previously reported by our laboratory ${ }^{22}$. The mean intensity and standard deviation of each fluorophore were measured, and background signal was removed by thresholding images to mean + standard deviation, corresponding to the top third brightest pixels 
131 in the Gaussian distribution. After thresholding, final measurements were made by taking the mean intensity of the remaining pixels. Values were normalized to the corresponding wild-type sex average.

\subsection{Activity-Based Protein Profiling}

Mice were euthanized via cervical dislocation and brains dissected and washed with ice cold lysis buffer $(0.25 \mathrm{M}$ sucrose, 20mM HEPES, 2mM DT'T, in deionized H2O) twice before dounce homogenization. Blood samples and brain tissues were processed using dounce homogenizer and placed on ice for 15 minutes. Tissue homogenates were centrifuged at $800 \mathrm{xg}$ for 5 minutes at $4^{\circ} \mathrm{C}$. Supernatants were separated to remove debris from unlysed tissue. Supernatants were centrifuged at 100,000xg for 45 minutes at $4^{\circ} \mathrm{C}$. Resulting pellet was resuspended in assay buffer $\left(20 \mathrm{mM}\right.$ HEPES in deionized $\left.\mathrm{H}_{2} \mathrm{O}\right)$. Proteome concentrations were normalized using Bio-Rad DC protein assay and treated with either HT-01 or FP-Rhodamine at 1uM final concentration for 30 minutes at $37^{\circ} \mathrm{C}$. The reaction was quenched using SDS-PAGE loading buffer. After separation by SDS-PAGE (10\% acrylamide), samples were visualized by in-gel fluorescence scanning using Chemidoc MP imaging system. Entire blots are depicted.

\subsection{Drug treatment and miniature osmotic pump surgery.}

KT-182 was synthesized in our laboratory as described $^{23}$. For acute studies, mice at 8 months of age were treated with KT-182 (2mg/kg), a dose shown to inhibit brain ABHD6 activity by more than 90\% when injected i.p., or with vehicle. Animals were monitored for 1 hour and then behavioral tests were conducted 4 hours post-injection to allow for full inhibition of ABHD6 by KT-182. All animals in the same cage were treated and tested at the same time. For semi-chronic studies, Alzet miniature osmotic pumps (1007D) were used over daily injections to reduce the stress and risk of infection due to daily handling and injection. Buprenorphine and lidocaine were used as analgesic care, and animals were anaesthetized with isoflurane. Pumps were filled with either vehicle or KT-182 and surgically implanted into female HdhQ200/200 and wild-type mice 7 days prior to 10 months of age. Drug was administered at a rate of $0.5 \mu \mathrm{l} /$ hour to achieve $2 \mathrm{mg} / \mathrm{kg} /$ day. Only mice undergoing surgery were housed together along with supplemental food mash and gel. Mice were checked on daily and after 5 days post-surgery, mice underwent behavioral studies. We found no significant effect of osmotic pump implantation on motor performance (untreated/HdhQ200/200 data versus vehicle; $\mathrm{p}=0.631$ for male, $\mathrm{p}=0.618$ for female). Thus, the effect of osmotic pump treatments was controlled as both WT and HdhQ200/200 mice received surgery and pumps. A small sample size was chosen due to the timeframe of experiments and initial intention to gather basic evidence.

\subsection{HdhQ200/200 behavioral studies.}


All animals undergoing testing were used from the same cages, of mixed genotype, and age-matched controls to minimize potential confounding factors. All behavioral studies were performed during the light phase of the light/dark cycle, and the tester was blinded by the conditions and genotype. Cages and equipment were thoroughly cleaned with 70\% ethanol and dried between trials. Grip strength was measured by recording the latency to fall when animals were placed on a wire cage top and then inverted. A maximum score of 90 seconds was used. Rotarod (Med Associates Inc., St. Albans, VT) testing included 7 consecutive trials, separated by 15-minute resting periods. The rotational speed used for testing trails began at $4 \mathrm{rpm}$ and increased to $40 \mathrm{rpm}$ for a maximum of 5 minutes, with an acceleration of $0.2 \mathrm{rpm}$ per every 4 seconds. Slow motor learning was measured by the sum of improvement in performance across all 7 trials ${ }^{24}$. The PhenoTyper (Noldus, Wageningen, the Netherlands) testing consisted of a 72-hour trial where animals were individually housed in a $30 \mathrm{~cm} \times 30 \mathrm{~cm}$ Plexiglas cage with ad libitum access to water and food. A vertically mounted camera continuously tracked movement and digital traces were analyzed in Ethovision XT 11. Data was taken from the dark phase of day 2 to ensure animals were habituated to the cage and undisturbed by any outside distractions. No exclusion criteria was set for behavioral data and no animals undergoing testing was excluded from the final data set.

\subsection{Statistical analyses}

Statistical analyses and graphs were generated using GraphPad PRISM 9 (San Diego, CA). Two-way ANOVA with a Bonferroni post-hoc analysis was used for measurements over time and a one-way ANOVA with Tukey's multiple comparison for averaged comparisons. Data were considered significant if $\mathrm{p}<0.05$.

\section{Results}

\subsection{HdhQ200/200 female and male mice do not exhibit overt psychiatric symptoms at 8 and 10}

\section{months of age.}

We previously reported that female HdhQ200/200 mice exhibited more pronounced deficits in locomotor and motor coordination at 8 months of age compared to male HdhQ200/200 mice ${ }^{7}$. To extend these results, we studied anxiety-like and depressive-like behaviors by measuring both thigmotaxis and time spent in the hidden zone of the home-cage during the dark phase using the PhenoTyper ${ }^{20,25}$. We found no difference in the time spent in the thigmotaxis or hidden zone by either male or female HdhQ200/200 mice when compared to their respective wild-type littermates (Supplementary Fig. 1A-F). Importantly, the frequency to enter either the thigmotaxis but not the hidden zone was significantly decreased for female HdhQ200/200 mice when compared to female wild-type littermates at 8 months of age (Supplementary Fig. 2A-F). This impairment was not detected when comparing male HdhQ200/200 and male W'T mice. Considering that the total times spent in each area was comparable for all genotypes, a decrease in frequency to enter the 
thigmotaxis can be attributed to decreased general locomotion in female HdhQ200/200 mice rather than evidence for an anxious or depressive phenotype, such as lack of motivation. Base on this premise, we focused the subsequent studies on locomotor and motor coordination impairments.

\subsection{At 8 months of age, acute ABHD6 inhibition rescued motor coordination deficits in female} HdhQ200/200 mice and improved motor coordination in male WT mice.

We studied the effect of acute ABHD6 inhibition on HdhQ200/200 mice and WT littermates of both sexes at 8 months of age, an age when motor behavior deficits are pronounced in female but absent in male

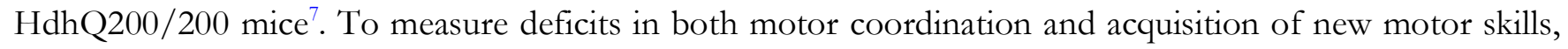
we measured the latency to fall off a rotarod over multiple trials ${ }^{24}$. As previously reported, female HdhQ200/200 performed 43\% worse than female W'T littermates $(\mathrm{p}=0.030)$ (Fig. 1A) ${ }^{7}$. To pharmacologically inhibit brain ABHD6 activity, we treated mice with KT-182, a brain-penetrant compound shown to reduce brain ABHD6 activity by more than 90\% ${ }^{23}$. Figures 1B-C show that KT-182 did not affect the performance of female W'T mice and yet greatly improved the performance of female HdhQ200/200 mice. Thus, acute treatment with KT-182 rescued this deficit in female HdhQ200/200 mice by $65 \%(\mathrm{p}<0.001)$ without influencing the rotarod performance of female WT mice (Fig. 1A). Interestingly, female HdhQ200/200 mice

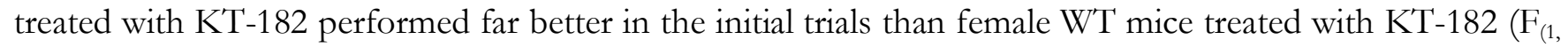
${ }_{95}=121.7, \mathrm{p}<0.001$ ) (Fig. 1C). These results show that acute ABHD6 inhibition did not affect motor coordination of female WT mice but rescued the motor coordination deficits caused by mtHTT in female HdhQ200/200 mice tested at 8 months of age.

To determine the effect of acute ABHD6 inhibition on the acquisition of new motor skills, we adopted a method in which slow motor learning was measured by the sum of improvement in performance across all trials $^{24}$. Figure 1D shows that slow motor learning was similar between female HdhQ200/200 and WT mice and that acute KT-182 treatment did not affect this measure. Thus, at 8 months of age, acute ABHD6 inhibition rescued motor coordination deficits in female HdhQ200/200 mice and this immediate therapeutic response remained evident across all 7 trials. By contrast, acute ABHD6 inhibition did not affect motor coordination in female W'T mice and did not affect slow motor learning in either group, showing that rescue was specific to deficits in motor coordination that develop in female HdhQ200/200 mice.

As previously reported, male HdhQ200/200 mice performed similarly to male WT littermates in motor coordination at 8 months of age (Supplementary Fig. 3A) ${ }^{7}$. Remarkably, KT-182 improved motor performance of male WT mice by 2 -fold $(\mathrm{p}=0.001)$ and yet did not affect the performance of male HdhQ200/200 mice (Supplementary Fig. 3A). The KT-182 response improved over all trials in male WT mice $\left(F_{(1,56)}=97.85, \mathrm{p}<0.01\right)$ (Supplementary Fig. 3B) but was absent in male HdhQ200/200 mice (Supplementary Fig. 3C). Accordingly, KT-182 also improved slow motor learning in male WT mice 
( $\mathrm{p}=0.005)$ but not in male HdhQ200/200 mice (Supplementary Fig. 3D). These results show that acute ABHD6 inhibition improves motor coordination in male W'T mice but does not affect motor coordination in male HdhQ200/200 mice tested at 8 months of age.

Based on this result, we focused our pilot study on female HdhQ200/200 mice. Previous experiments suggested that acute treatment of female HdhQ200/200 mice at 10 months of age with KT-182 did not affect their pronounced deficit in motor coordination (data not shown), indicating that a prolonged treatment approach at a greater symptomatic stage might be required to test the effects of ABHD6 inhibition.

3.3 Striatal ABHD6 expression in female HdhQ200/200 mice remained unchanged at 8 months of age but was reduced by 10 months of age.

To determine if ABHD6 expression changes in the striatum of HdhQ200/200 mice, we used a purified rabbit polyclonal antibody that recognizes a 38 amino acid epitope in MABHD6 and had been previously validated by our laboratory ${ }^{14}$. To further rigorously validate this new affinity-purified antibody, we immunostained brain coronal sections from W'T and ABHD6-knockout mice ${ }^{19}$. Figures 2A-B illustrate ABHD6 expression in mouse dorsal striatum and suggest enhanced expression in select striatal cells. Figure 2C validates the selectivity of the antibody by confirming the absence of immunofluorescence signal in sections from ABHD6knockout mice.

Motor coordination is significantly impaired in female HdhQ200/200 mice at both 8 and 10 months of age $^{7}$. To determine if ABHD6 expression is also affected by HD pathogenesis, we immunostained brain coronal sections of female HdhQ200/200 mice and W'T littermates at 8 and 10 months of age and measured striatal ABHD6 expression levels with semi-quantitative immunohistochemistry (sqIHC) as previously described by our laboratory ${ }^{7,18,22}$. We found no difference in ABHD6 expression between female HdhQ200/200 and W'T striatum at 8 months of age but a significant 40\% decrease in expression at 10 months of age $\left(\mathrm{F}_{(1,15)}=5.59, \mathrm{p}=0.032\right)$ (Fig. 2D). Figures $2 \mathrm{E}-\mathrm{H}$ show representative images of IHC staining of ABHD6 expression in the striatum of female HdhQ200/200 and WT mice at 8 and 10 months of age and illustrates reduced ABHD6 expression in female HdhQ200/200 at 10 months of age. Thus, striatal ABHD6 expression in female HdhQ200/200 mice decreases late in the disease (i.e. after symptom onset and before end-stage).

\subsection{Semi-chronic treatment with KT-182 safely blocked brain ABHD6 activity.}

The reduction in striatal ABHD6 expression in female HdhQ200/200 mice at 10 months of age suggests that a semi-chronic treatment with KT-182 is preferable. Thus, we sought to use miniature osmotic pumps as a semi-chronic delivery system. Mice were surgically implanted with an Alzet ${ }^{\circledR}$ miniature osmotic pump containing KT-182 that enables constant drug delivery for 7 days. While it is known that KT-182 crosses the 
blood-brain-barrier ${ }^{23}$, its effectiveness in inhibiting ABHD6 following administration via osmotic pumps has yet to be determined. We validated this approach using competitive activity-based protein profiling (ABPP) with the fluorescence probe HT- $01^{26}$. Brain ABHD6 activity was drastically reduced in W'T mice after 7 days of KT-182 administration (Fig. 3A). Inhibition appeared to be selective to ABHD6, as activities of other lipid-degrading enzymes (such as fatty acid amid hydrolase (FAAH) and diacylglycerol lipase (DAGL)) were not inhibited in drug-treated animals. Furthermore, ABHD6 activity in blood samples was undetectable in KT-182 treated mice (data not shown). Importantly, female HdhQ200/200 mice and WT littermates carrying osmotic pumps with vehicle or KT-182 for 7 days at 10 months of age did not show overt side effects. This treatment regimen did not affect the overall weight or grip strength in mice (Fig. 3B-C), nor were any other behavioral changes observed. These results show that osmotic pump delivery of KT-182 for 7 days achieves near-complete inhibition of ABHD6 in the brain without triggering overt side effects in WT and symptomatic HdhQ200/200 female mice.

\subsection{At 10 months of age, semi-chronic ABHD6 inhibition rescued motor coordination deficit in} female HdhQ200/200 mice.

As previously reported, motor coordination was decreased by 31\% in female HdhQ200/200 mice compared to W'T littermates at 10 months of age $(\mathrm{p}=0.046)$ (Fig. 4A) ${ }^{7}$. Figures 4A-C show that KT-182 did not affect the motor performance of female W'T mice but greatly improved the performance of female HdhQ200/200 mice $\left(\mathrm{F}_{(1,70)}=33.53, \mathrm{p}<0.001\right)$. Thus, semi-chronic treatment with KT-182 rescued this deficit selectively in symptomatic HdhQ200/200 mice by 53\% to be comparable to WT levels ( $\mathrm{p}=0.019)$ (Fig. 4A). Slow motor learning was similar between female HdhQ200/200 mice and WT littermates treated with vehicle, as previously reported ${ }^{7}$, and semi-chronic inhibition of ABHD6 did not affect this (Fig. 4D). Thus, at 10 months of age, semi-chronic ABHD6 inhibition rescued motor coordination deficits in female HdhQ200/200 mice but did not affect motor coordination in female W'T mice or slow motor learning in either group, showing that rescue at 10 months of age was specific to deficits in motor coordination that develop in this HD mouse model.

\subsection{At 10 months of age, semi-chronic inhibition of ABHD6 did not affect spontaneous locomotion} of female HdhQ200/200 mice and wild-type littermates.

To determine if semi-chronic inhibition of ABHD6 might affect general locomotion, we measured spontaneous locomotion of female WT and HdhQ200/200 mice using the PhenoTyper. As previously reported, female HdhQ200/200 mice and WT littermates exhibited similar exploratory behavior of a novel environment (as measured by the distance traveled during the first hour of being placed into the chamber) ${ }^{7,27}$ (Fig. 5A). As expected, female HdhQ200/200 mice exhibited reduced spontaneous locomotion during the 
dark phase (when mice are more active) compared to female WT mice $(p=0.040)$, yet similar spontaneous locomotion during the light phase (when mice are less active) compared to female WT mice at 10 months of age (Fig. 5B-C). Semi-chronic inhibition of ABHD6 did not affect these behaviors in both groups during either phase (Fig. 5A-C). These results confirm our previous study showing that female HdhQ200/200 mice are less active during the dark compared to female W'T mice and show that spontaneous locomotion in both groups is unaffected by semi-chronic inhibition of ABHD6.

\section{Discussion}

Our current study shows preliminary data that the pharmacological inhibition of ABHD6 through osmotic pump delivery rescues motor coordination deficits in female HdhQ200/200 mice, a mouse model of HD that exhibits robust early onset deficits in multiple motor behaviors compared to female WT littermates. Markedly, ABHD6 inhibition rescued deficits in motor coordination without affecting other deficits in locomotion exhibited by female HdhQ200/200 mice. Furthermore, ABHD6 inhibition did not cause overt side effects (i.e., change in overall weight, grip strength, novel environment exploration and spontaneous locomotion during the light phase, or changes in observed behaviors), suggesting a promising safety profile for this class of compounds. Using a validated rabbit polyclonal antibody that recognizes ABHD6 in mouse tissue, we found that ABHD6 expression remains unchanged in female HdhQ200/200 mice at 8 months of age compared to WT littermates but is reduced by 10 months of age. The interaction that we report here suggests several possible molecular mechanisms that could link the reduction in striatal ABHD6 expression and selective rescue of motor coordination deficits in female HdhQ200/200 mice.

The expression and function of many genes and proteins change as a result of mtHTT during HD pathogenesis $^{28}$, including several other serine hydrolases that control the eCB signaling system ${ }^{29,30}$. The activity of FAAH, DAGL and N-acylphosphatidylethanolamine-specific phospholipase D change in the striatum of R6/2 mice in relation to disease progression, whereas monoacylglycerol lipase (MAGL) activity is unchanged compared to WT mice ${ }^{31,32}$. In HD43 cells in culture, inducible expression of mtHTT reduces FAAH activity and increases MAGL activity ${ }^{31}$. In primary mouse striatal astrocytes, mtHTT expression increases MAGL mRNA levels without changing $\mathrm{FAAH}^{33}$. Other examples of changes in serine hydrolase activities associated with HD pathogenesis include acetylcholinesterase ${ }^{34}$, palmitoyl acyltransferase ${ }^{35,36}$ and transglutaminase $2^{37}$. Here, we report the downregulation of ABHD6 expression in female HdhQ200/200 mice, adding a new serine hydrolase to the list of eCB enzymes affected by HD pathogenesis.

Considering the expression pattern of ABHD6 in the striatum and range of signaling mechanisms that this enzyme modulates, reduced expression of ABHD6 in HdhQ200/200 striatum detected at 10 months of age is likely to affect multiple aspects of neuronal function beyond its ability to control eCB signaling, including 
post-synaptic GABAergic currents and trafficking of AMPA receptors to the plasma membrane ${ }^{12}$. We show that ABHD6 is expressed at higher levels in select striatal cells with neuronal-like morphology, which agrees with the single-cell RNA-seq analyses of mouse striatum showing that ABHD6 mRNA expression is abundant in interneurons ${ }^{13}$ (http://www.brainrnaseq.org/). Considering the role of striatal interneurons in the control of cortico-striatal projections and how their functions are impaired by HD pathogenesis ${ }^{38-40}$, ABHD6 downregulation might contribute to symptoms measured in HdhQ200/200 mice at 10 months of age by disrupting the functionality of the cortico-striatal projections.

Few therapeutic approaches designed to target serine hydrolases have been tested in HD mouse models. Promising results have been reported for the pharmacological inhibition of the serine protease HTRA2 ${ }^{41}$ and transglutaminase $2^{37}$, and a recent study validated MAGL expressed by astrocytes as a potential therapeutic target for $\mathrm{HD}^{33}$. Our results add to this body of knowledge by showing that ABHD6 inhibition rescued a specific, early onset symptom - motor coordination - in female HdhQ200/200 mice, providing a new therapeutic approach for the treatment of select symptoms associated with HD. In addition, due to the lack of depressive or anxious phenotype in the HdhQ200/200 model, we can conclude that this rescue was select for motor behaviors and not through motivation or other psychiatric symptoms. Our pilot study also emphasizes the need to test and optimize dosing regimens of AHBD6 inhibition as a function of disease progression as we found that semi-chronic, but not acute, inhibition of ABHD6 was required to rescue deficits in motor coordination in HdhQ200/200 mice at 10 months of age.

We can draw at least two potential molecular and cellular mechanisms by which ABHD6 inhibition might rescue motor coordination in female HdhQ200/200 mice. First, serine hydrolases are one of the largest known classes of enzymes, each with remarkably different enzymatic activities performed by the common defining molecular characteristic of a nucleophilic serine in the active site ${ }^{42}$. The pocket formed by the catalytic site of ABHD6 hydrolyzes select monoacylglycerols (including the eCB 2-arachidonoyl glycerol (2-AG)), and carbamate-based inhibitors (such as KT-182) block the hydrolyzing activity through a covalent modification of the catalytic Ser148 $23,43-45$. Thus, inhibition of the ABHD6 catalytic triad mediated-activity favors 2-AG accumulation in post-synaptic compartments of active neurons, which in turn enhances both $\mathrm{CB}_{1} \mathrm{R}$-mediated presynaptic inhibition of neurotransmitters release and post-synaptic GABAergic currents ${ }^{12}$. By contrast, the ability of ABHD6 to control the number of AMPA receptors trafficking to the plasma membrane does not involve the catalytic triad ${ }^{46}$, and thus the rescue of motor coordination deficit in HdhQ200/200 by KT-182 is unlikely to involve this AMPA receptor-dependent mechanism. Second, at 8 months of age, when striatal ABHD6 expression is similar in W'T and HdhQ200/200 mice, acute ABHD6 inhibition enhanced rotarod coordination in female HdhQ200/200 mice but not in W'T littermates. This result suggests the existence of a molecular mechanism, or lack of molecular mechanism, that is present in female HdhQ200/200 mice and not 
in female W'T mice that enables ABHD6 inhibition to improve motor coordination only in female HdhQ200/200 mice. Thus, our study suggests the following picture whereby inhibition of striatal ABHD6 influences the function of defective cortico-striatal pathways in female HdhQ200/200 mice in such a way to rescue deficits in motor coordination ${ }^{38-40}$.

361 Our preliminary studies on acute ABHD6 inhibition led to a remarkable increase in motor coordination measured in male WT mice at 8 months of age but not male HdhQ200/200 mice. While the molecular mechanism of this response remains to be studied, two conclusions can be drawn from this result: male WT mice express molecular components that enhance the efficacy of acute ABHD6 inhibition to regulate motor coordination, and these molecular components are absent in male HdhQ200/200 mice at 8 months of age. Further studies are needed to tease apart this difference.

Few treatments are available to help HD patients manage the multiple debilitating symptoms that arise as a function of disease progression. Here, we provide the first preliminary evidence that pharmacological inhibition of ABHD6 selectively rescues deficits in motor coordination modeled by female HdhQ200/200 mice. Our results suggest a mechanistic link between the reduction in striatal ABHD6 expression and selective rescue of motor coordination deficits in female HdhQ200/200 mice. Thus, ABHD6 inhibitors represent promising pharmacological tools to unravel the molecular mechanisms involved in motor coordination deficits in neurological diseases and could lead to the development of a new therapeutic modality to treat deficits in locomotor behaviors associated with HD.

Acknowledgments: This work was supported by the National Institutes of Health (T32 MH015442 to JK. C., DA043571 to K-L. H., DA037673 and DA011322 to K.M. and DA026430 to N.S.).

Author contributions: JKC and NS designed studies and wrote manuscript. JKC and KV performed behavioral tests. JKC and RW performed imaging studies. MS performed assays. KLS and KM provided expertise support. NS supervised project.

Figure 1: At 8 months of age, acute inhibition of ABHD6 rescued motor coordination deficits in female HdhQ200/200 mice.

Motor coordination was measured using rotarod analysis in wild type (WT) and HdhQ200/200 mice (8 months of age) that were acutely treated with either vehicle or KT-182. A. Analysis of the average latency to fall showed that KT-182 did not affect motor coordination in female WT mice but significantly rescued motor coordination in female HdhQ200/200 mice. Statistical analysis: One-way ANOVA analysis (multiple comparisons using Tukey) indicated a 49\% reduction in latency to fall in HdhQ200/200 mice compared to 
WT mice and a 58\% rescue of latency to fall in HdhQ200/200 mice with KT-182. B-C. KT-182 treatment did not affect latency to fall in (B) female WT mice and enhanced latency to fall in (C) female HdhQ200/200 mice across all trials. Statistical analysis: Two-way ANOVA with a Bonferroni post-hoc analysis indicated significant rescue of motor coordination deficit in HdhQ200/200 by KT-182 compared to vehicle-treated HdhQ200/200 mice. D. Slow motor learning was comparable between female HdhQ200/200 mice and WT littermates, and unaffected by acute treatment of KT-182. $\mathrm{N}=6-11$ mice per condition, error bars represent S.E.M. and ${ }^{*} \mathrm{p}<0.05,{ }^{* *} \mathrm{p}<0.01,{ }^{* * *} \mathrm{p}<0.001$.

Figure 2: Reduced striatal ABHD6 expression in female HdhQ200/200 mice compared to wild-type mice at 10 months of age.

Immunohistochemistry (IHC) analysis of ABHD6 expression in mouse striatum using rabbit polyclonal antibodies that recognize mABHD6 showed no change between wild-type (WT) and HdhQ200/200 female mice at 8 months of age but a decrease in ABHD6 expression in HdhQ200/200 mice at 10 months of age. A. Representative IHC images show discreet ABHD6 expression in W'T mouse striatum (12 weeks of age). Scale bar $=50 \mathrm{~mm}$. B. Representative IHC images show higher expression of ABHD6 in select striatal cells. Scale bar $=25 \mathrm{~mm}$. C. Validation of antibody selectivity via IHC staining showed no immunostaining in Abbd6 ${ }^{-1}$ mouse striatum (12 weeks of age). Scale bar $=25 \mathrm{~mm}$. D. Semi-quantitative IHC analysis of ABHD6 expression in WT and HdhQ200/200 showed no change at 8 months of age but significant decrease at 10 months of age in HdhQ200/200 mice. Statistical analysis: Two-way ANOVA with a Bonferroni post-hoc analysis indicated no difference at 8 months of age but a $40 \%$ decrease at 10 months of age. E-H. Representative striatal ABHD6 IHC staining at (E) 8 months of age in female WT and (F) HdhQ200/200 mice and $(\mathrm{G}) 10$ months of age in female W'T and $(\mathrm{H}) \mathrm{HdhQ} 200 / 200$ mice. Scale bar $=50 \mathrm{~mm} . \mathrm{N}=4-5$ mice per condition, error bars represent S.E.M. and ${ }^{*} \mathrm{p}<0.05$.

Figure 3: Semi-chronic, subcutaneous, osmotic pump delivery of KT-182 resulted in on-target inhibition of brain ABHD6 activity and did not affect overall body weight or grip strength of wildtype and HdhQ200/200 mice at 10 months of age.

Osmotic pumps delivering KT-182 were implanted subcutaneously (s.c.) in wild-type (WT) mice (12 weeks of age) for 10 days and ABHD6 activity was measured by activity base protein profiling. A. 10 days s.c. osmotic delivery of KT-182 (lanes K1, K2 and K3) inhibited brain ABHD6 activity compared to vehicle-treated animals (lanes VEH1, VEH2 and VEH3) without affecting DAGLa, DAGLb and FAAH activity. $\mathrm{N}=3$ mice per treatment. B-C. Semi-chronic inhibition of ABHD6 did not affect (B) weight loss or (C) grip strength as measured by the inverted wire test of HdhQ200/200 mice compared to WT mice (10 months of age). 
421 Statistical analysis: One-way ANOVA analysis (multiple comparisons using Tukey) indicated significant 422 reduction in overall body weight of HdhQ200/200 mice compared to WT and no significant difference in 423 grip strength. $\mathrm{N}=5-8$ mice per condition, error bars represent S.E.M. and ${ }^{*} \mathrm{p}<0.05$.

424

425

426

427

428

429

430

431

432

433

434

435

436

437

438

439

440

441

442

443

444

445

446

447

448

449

450

451

Figure 4: Semi-chronic inhibition of ABHD6 rescued motor coordination deficits in HdhQ200/200 mice at 10 months of age.

Motor coordination was measured in female wild type (WT) and HdhQ200/200 mice (10 months of age) that were implanted with osmotic pumps delivering either vehicle or KT-182. A. Analysis of average latency to fall from the rotarod measured across 7 trials showed that KT-182 did not affect motor coordination in W'T mice but significantly rescued motor coordination in HdhQ200/200 mice. Statistical analysis: One-way ANOVA analysis (multiple comparisons using Tukey) indicated a 31\% reduction in latency to fall in HdhQ200/200 mice compared to WT mice and a 53\% rescue of latency to fall in HdhQ200/200 mice when treated with KT182. B. KT-182 treatment did not affect latency to fall in W'T mice but C. enhanced latency to fall in HdhQ200/200 mice. Statistical analysis: Two-way ANOVA analysis with Bonferroni post-hoc analysis indicated significant rescue of motor coordination deficits in HdhQ200/200 by KT-182 compared to vehicletreated HdhQ200/200 mice. D. Slow motor learning was comparable between HdhQ200/200 mice and W'T littermates and not affected by treatment with KT-182. $\mathrm{N}=6-7$ mice per condition, error bars represent S.E.M. and ${ }^{*} \mathrm{p}<0.05,{ }^{*} \mathrm{p}<0.01$.

Figure 5: Semi-chronic inhibition of ABHD6 did not affect spontaneous locomotion of wild-type and HdhQ200/200 mice at 10 months of age.

Spontaneous locomotion (meters/hour) was measured in female wild type (W'T) and HdhQ200/200 mice (10 months of age) that were implanted with osmotic pumps delivering either vehicle or KT-182 using PhenoTyper chambers. A. Distance traveled during the first hour of entering the PhenoTyper chamber indicates exploration of a novel environment and was unaffected by genotype or KT-182 treatment. Distance traveled measured during the B. dark phase (high activity, 12 hours) and $\mathbf{C}$. light phase (low activity, 12 hours). Statistical analysis: One-way ANOVA analysis (multiple comparisons using Tukey) indicated a 41\% reduction in spontaneous locomotion in HdhQ200/200 compared to WT measured during the dark phase but no difference between vehicle and KT-182. $\mathrm{N}=6$ mice per condition, error bars represent S.E.M. and *p $<0.05$. 
Landles, C. \& Bates, G. P. Huntingtin and the molecular pathogenesis of Huntington's disease. Fourth in molecular medicine review series. EMBO Rep 5, 958-963, doi:10.1038/sj.embor.7400250 (2004).

Ross, C. A. \& Tabrizi, S. J. Huntington's disease: from molecular pathogenesis to clinical treatment. Lancet Neurol 10, 83-98, doi:10.1016/S1474-4422(10)70245-3 (2011).

Roze, E. et al. in Frontiers in neuroanatomy Vol. 5 (Frontiers Media SA, 2011).

Walker, F. O. in Lancet Vol. 369 218-228 (Elsevier, 2007).

Lin, C.-H. et al. in Human Molecular Genetics Vol. 10 137-144 (Oxford Univ Press, 2001).

Pouladi, M. A., Morton, A. J. \& Hayden, M. R. Choosing an animal model for the study of Huntington's disease. Nat Rev Neurosci 14, 708-721, doi:10.1038/nrn3570 (2013).

Cao, J. K., Viray, K., Zweifel, L. \& Stella, N. Sex-dependent impaired locomotion and motor coordination in the HdhQ200/200 mouse model of Huntington's Disease. Neurobiology of disease 132, 104607 (2019).

Heng, M. Y. et al. Early autophagic response in a novel knock-in model of Huntington disease. Hum Mol Genet 19, 3702-3720, doi:10.1093/hmg/ddq285 (2010).

Pearl, J. R. et al. Enhanced retinal responses in Huntington's disease patients. Journal of Huntington's disease 6, 237-247 (2017). Zielonka, D. et al. The influence of gender on phenotype and disease progression in patients with Huntington's disease. Parkinsonism \& related disorders 19, 192-197 (2013).

Zielonka, D. \& Stawinska-Witoszynska, B. Gender Differences in Non-sex Linked Disorders: Insights From Huntington\&apos;s Disease. Frontiers in Neurology 11, 859-855, doi:10.3389/fneur.2020.00571 (2020).

Cao, J. K., Kaplan, J. \& Stella, N. ABHD6: Its Place in Endocannabinoid Signaling and Beyond. Trends in pharmacological sciences (2019). doi:10.1016/j.celrep.2016.06.059 (2016)

Marrs, W. R. et al. The serine hydrolase ABHD6 controls the accumulation and efficacy of 2-AG at cannabinoid receptors. Nat Neurosci 13, 951-957, doi:10.1038/nn.2601 (2010).

Tchantchou, F. \& Zhang, Y. Selective Inhibition of Alpha/Beta-Hydrolase Domain 6 Attenuates Neurodegeneration, Alleviates Blood Brain Barrier Breakdown, and Improves Functional Recovery in a Mouse Model of Traumatic Brain Injury. Journal of Neurotrauma 30, 565-579, doi:10.1089/neu.2012.2647 (2013).

Wen, J., Ribeiro, R., Tanaka, M. \& Zhang, Y. Activation of CB2 receptor is required for the therapeutic effect of ABHD6 inhibition in experimental autoimmune encephalomyelitis. Neuropharmacology 99, 196-209, doi:10.1016/j.neuropharm.2015.07.010 (2015).

Manterola, A. et al. Deregulation of the endocannabinoid system and therapeutic potential of ABHD6 blockade in the cuprizone model of demyelination. Biochemical pharmacology, doi:10.1016/j.bcp.2018.07.042 (2018). mice. Neuron 83, 361-371, doi:10.1016/j.neuron.2014.06.030 (2014).

Van Esbroeck, A. C. M. et al. Identification of $\alpha$, $\beta$-hydrolase domain containing protein 6 as a diacylglycerol lipase in Neuro-2a cells. Frontiers in molecular neuroscience 12, 286 (2019).

Cao, J. K., Detloff, P. J., Gardner, R. G. \& Stella, N. Sex-dependent behavioral impairments in the HdhQ350/+ mouse line. Behav Brain Res 337, 34-45, doi:10.1016/j.bbr.2017.09.026 (2018).

Cao, J. K., Viray, K., Zweifel, L. \& Stella, N. Sex-dependent impaired locomotion and motor coordination in the HdhQ200/200 mouse model of Huntington\&apos;s Disease. Neurobiology of Disease 132, 104607, doi:10.1016/j.nbd.2019.104607 (2019).

Horne, E. A. et al. Downregulation of cannabinoid receptor 1 from neuropeptide $Y$ interneurons in the basal ganglia of patients with Huntington's disease and mouse models. Eur J Neurosci 37, 429-440, doi:10.1111/ejn.12045 (2013).

Hsu, K.-L. et al. Discovery and optimization of piperidyl-1, 2, 3-triazole ureas as potent, selective, and in vivo-active inhibitors of $\alpha / \beta$-hydrolase domain containing 6 (ABHD6). Journal of medicinal chemistry 56, 8270-8279 (2013).

Costa, R. M., Cohen, D. \& Nicolelis, M. A. L. in Current Biology Vol. 14 1124-1134 (2004).

Steele, A. D., Jackson, W. S., King, O. D. \& Lindquist, S. The power of automated high-resolution behavior analysis revealed by its application to mouse models of Huntington's and prion diseases. Proc Natl Acad Sci U S A 104, 1983-1988, doi:0610779104 [pii] 1073/pnas.0610779104 (2007). Hsu, K.-L. et al. DAGL $\beta$ inhibition perturbs a lipid network involved in macrophage inflammatory responses. Nature chemical biology 8, 999 (2012).

Steinbach, J. M., Garza, E. T. \& Ryan, B. C. Novel Object Exploration as a Potential Assay for Higher Order Repetitive Behaviors in Mice. J Vis Exp, doi:10.3791/54324 (2016). 9. 1259-1271, doi:ddd139 [pii] (2000). Friedman, D., French, J. A. \& Maccarrone, M. Safety, efficacy, and mechanisms of action of cannabinoids in neurological disorders. The Lancet Neurology 18, 504-512 (2019). Aymerich, M. S. et al. Cannabinoid pharmacology/the
system. Biochemical pharmacology 157, 67-84 (2018). Bari, M. et al. In vitro and in vivo models of $\mathrm{H}$ untington's disease show alterations in the endocannabinoid system. The FEBS journal 280, 3376-3388 (2013). Blazquez, C. et al. Loss of striatal type 1 cannabinoid receptors is a key pathogenic factor in Huntington's disease. Brain 134, 119136, doi:awq278 [pii] 
33 Ruiz-Calvo, A. et al. Astroglial monoacylglycerol lipase controls mutant huntingtin-induced damage of striatal neurons. Neuropharmacology (2019).

34 Ferrante, R. J., Beal, M. F., Kowall, N. W., Richardson Jr, E. P. \& Martin, J. B. Sparing of acetylcholinesterase-containing striatal neurons in Huntington's disease. Brain research 411, 162-166 (1987). Sanders, S. S. \& Hayden, M. R. (Portland Press Ltd., 2015). Rush, D. B., Leon, R. T., McCollum, M. H., Treu, R. W. \& Wei, J. Palmitoylation and trafficking of GAD65 are impaired in a cellular model of Huntington's disease. Biochemical Journal 442, 39-48 (2012).

Prime, M. E. et al. Discovery and structure-activity relationship of potent and selective covalent inhibitors of transglutaminase 2 for Huntington's disease. Journal of medicinal chemistry 55, 1021-1046 (2012). Cicchetti, F., Prensa, L. a., Wu, Y. \& Parent, A. Chemical anatomy of striatal interneurons in normal individuals and in patients with Huntington's disease. Brain research reviews 34, 80-101 (2000).

39 Cepeda, C. et al. Multiple sources of striatal inhibition are differentially affected in Huntington's disease mouse models. Journal of Neuroscience 33, 7393-7406 (2013).

40 Reiner, A. \& Deng, Y. P. Disrupted striatal neuron inputs and outputs in Huntington's disease. CNS neuroscience \& therapeutics 24, 250-280 (2018).

41 Goffredo, D. et al. Prevention of cytosolic IAPs degradation: a potential pharmacological target in Huntington's Disease. Pharmacological research 52, 140-150 (2005).

42 Liu, Y., Patricelli, M. P. \& Cravatt, B. F. Activity-based protein profiling: the serine hydrolases. Proc Natl Acad Sci U S A 96, 1469414699 (1999).

43 Blankman, J. L., Simon, G. M. \& Cravatt, B. F. A comprehensive profile of brain enzymes that hydrolyze the endocannabinoid 2arachidonoylglycerol. Chem Biol 14, 1347-1356 (2007).

$44 \quad$ Kaczor, A. A. et al. Comparative molecular field analysis and molecular dynamics studies of $\alpha / \beta$ hydrolase domain containing 6 (ABHD6) inhibitors. Journal of molecular modeling 21, 250, doi:10.1007/s00894-015-2789-8 (2015).

45 Bowman, A. L. \& Makriyannis, A. Highly Predictive Ligand-based Pharmacophore and Homology Models of ABHD6. Chemical biology \& drug design 81, 382-388 (2013).

46 Wei, M. et al. $\alpha / \beta$-Hydrolase domain-containing 6 (ABHD6) negatively regulates the surface delivery and synaptic function of AMPA receptors. Proceedings of the National Academy of Sciences 113, E2695-E2704 (2016). 
Figures

A
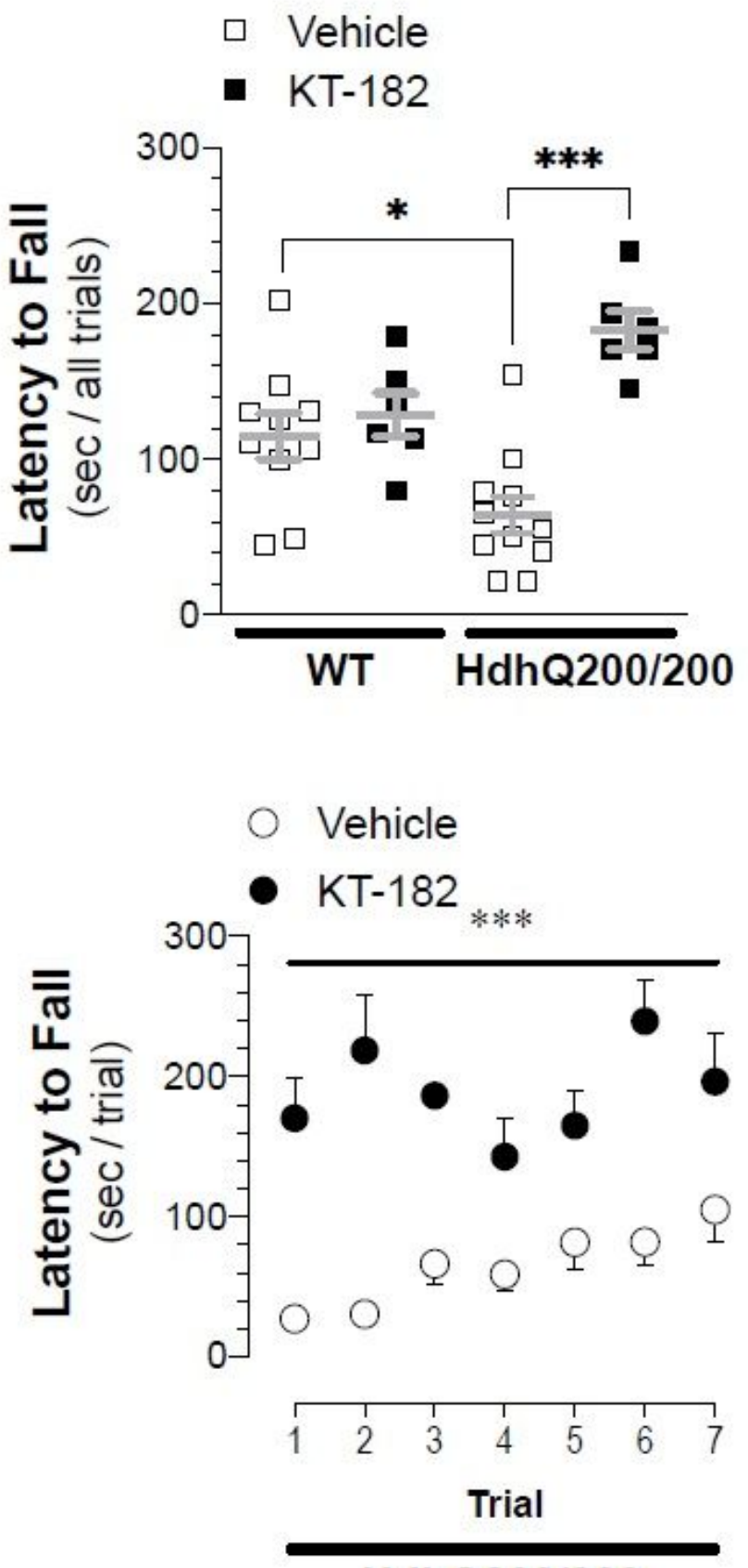

HdhQ200/200
B

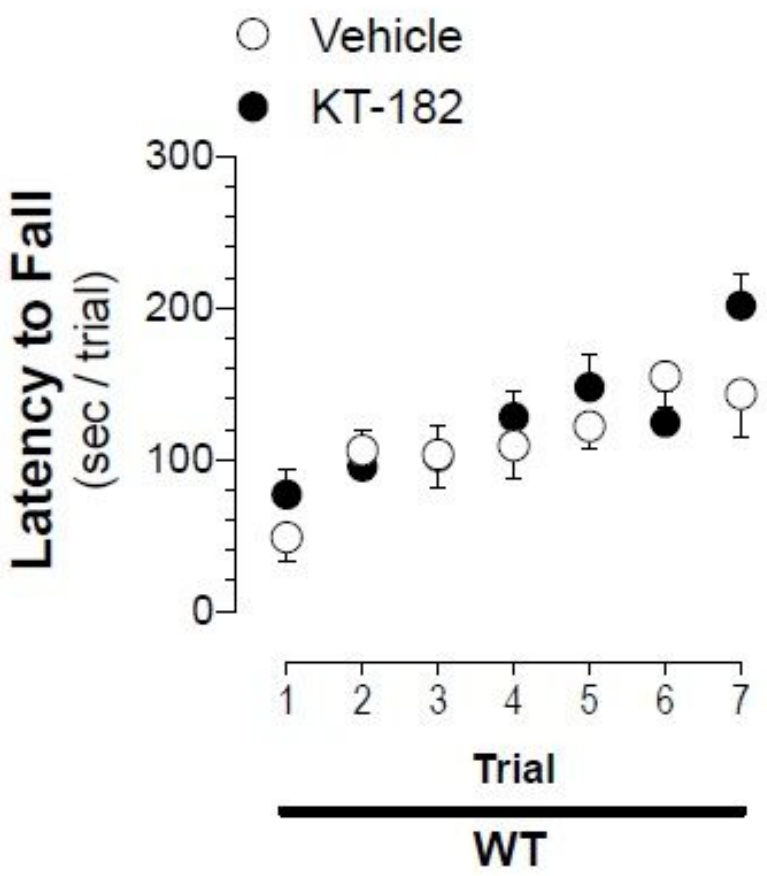

D

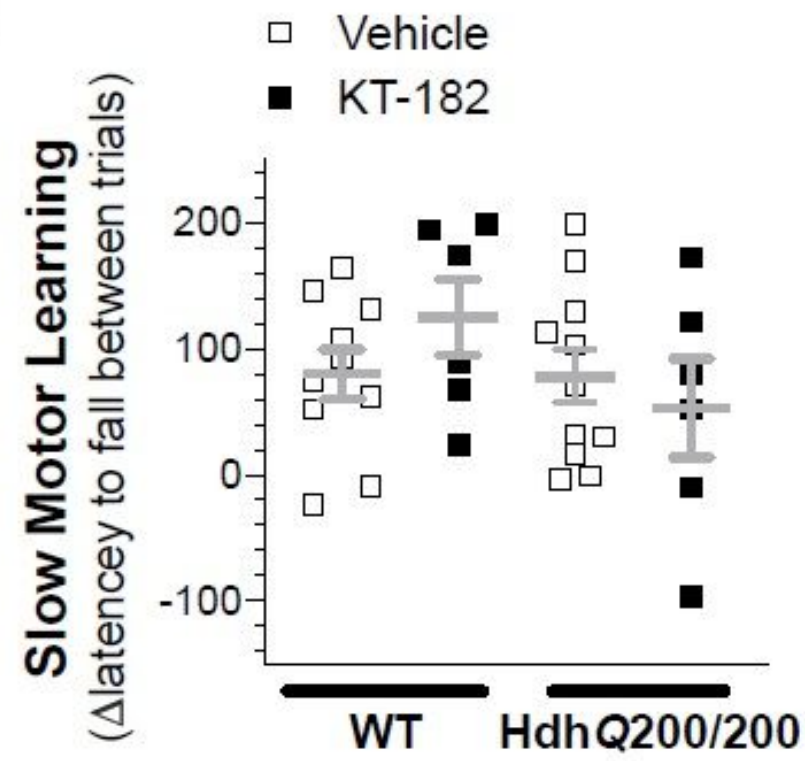

Figure 1

At 8 months of age, acute inhibition of ABHD6 rescued motor coordination deficits in female HdhQ200/200 mice. Motor coordination was measured using rotarod analysis in wild type (WT) and HdhQ200/200 mice (8 months of age) that were acutely treated with either vehicle or KT-182. A. Analysis of the average latency to fall showed that KT-182 did not affect motor coordination in female WT mice but significantly rescued motor coordination in female HdhQ200/200 mice. Statistical analysis: One-way 
ANOVA analysis (multiple comparisons using Tukey) indicated a $49 \%$ reduction in latency to fall in HdhQ200/200 mice compared to WT mice and a 58\% rescue of latency to fall in HdhQ200/200 mice with KT-182. B-C. KT-182 treatment did not affect latency to fall in (B) female WT mice and enhanced latency to fall in (C) female HdhQ200/200 mice across all trials. Statistical analysis: Two-way ANOVA with a Bonferroni post-hoc analysis indicated significant rescue of motor coordination deficit in HdhQ200/200 by KT-182 compared to vehicle-treated HdhQ200/200 mice. D. Slow motor learning was comparable between female HdhQ200/200 mice and WT littermates, and unaffected by acute treatment of KT-182. N = 6-11 mice per condition, error bars represent S.E.M. and ${ }^{\star} p<0.05,{ }^{\star \star} p<0.01,{ }^{\star \star \star} p<0.001$. 

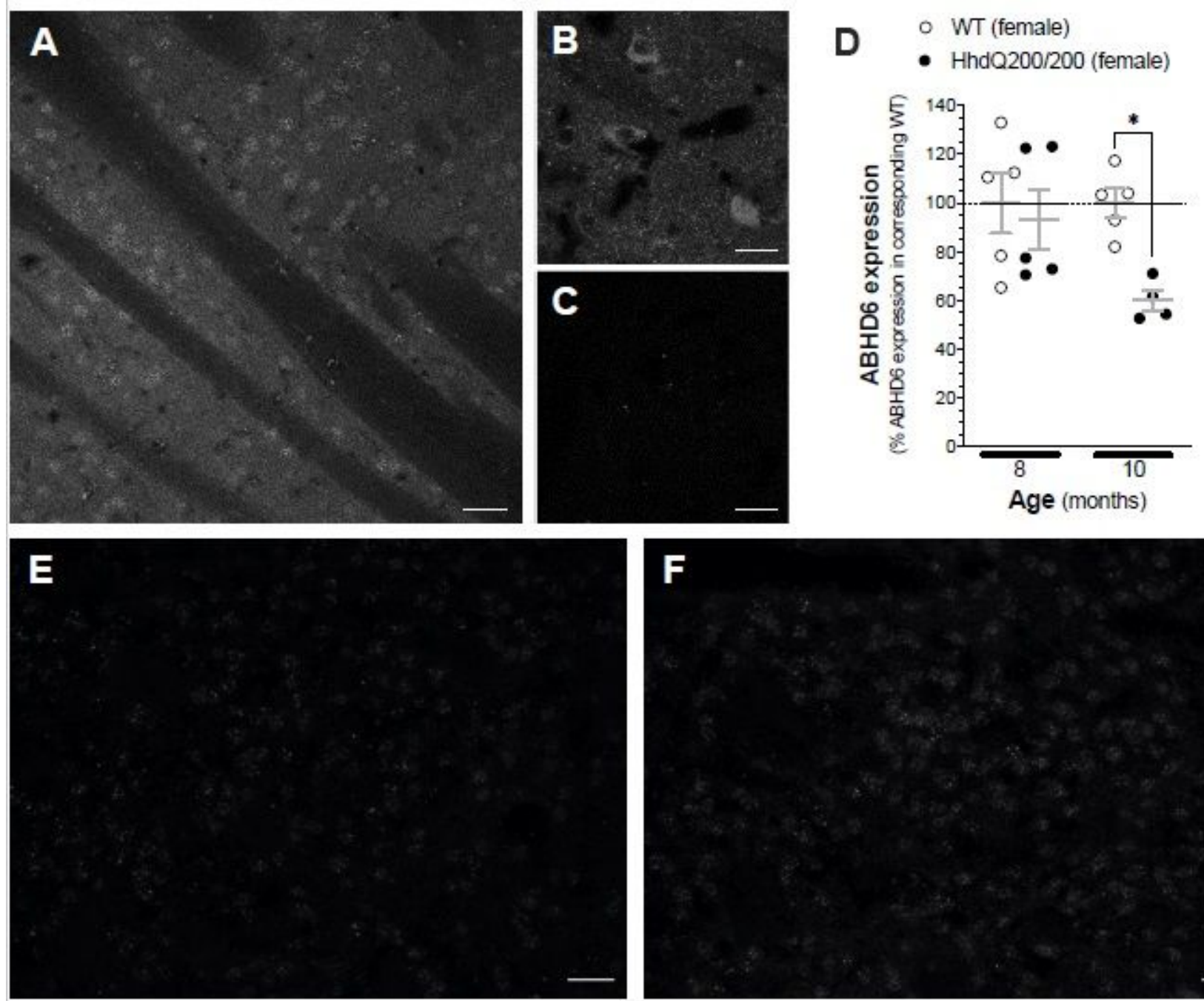

$\mathbf{F}$

G

H

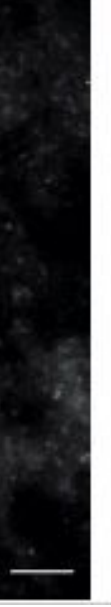

Figure 2

Reduced striatal ABHD6 expression in female HdhQ200/200 mice compared to wild-type mice at 10 months of age. Immunohistochemistry (IHC) analysis of ABHD6 expression in mouse striatum using rabbit polyclonal antibodies that recognize mABHD6 showed no change between wild-type (WT) and HdhQ200/200 female mice at 8 months of age but a decrease in ABHD6 expression in HdhQ200/200 mice at 10 months of age. A. Representative IHC images show discreet ABHD6 expression in WT mouse 
striatum (12 weeks of age). Scale bar $=50 \mathrm{~mm}$. B. Representative IHC images show higher expression of ABHD6 in select striatal cells. Scale bar $=25 \mathrm{~mm}$. C. Validation of antibody selectivity via IHC staining showed no immunostaining in Abhd6-/- mouse striatum (12 weeks of age). Scale bar $=25 \mathrm{~mm}$. D. Semiquantitative IHC analysis of ABHD6 expression in WT and HdhQ200/200 showed no change at 8 months of age but significant decrease at 10 months of age in HdhQ200/200 mice. Statistical analysis: Two-way ANOVA with a Bonferroni post-hoc analysis indicated no difference at 8 months of age but a $40 \%$ decrease at 10 months of age. E-H. Representative striatal ABHD6 IHC staining at (E) 8 months of age in female WT and (F) HdhQ200/200 mice and (G) 10 months of age in female WT and (H) HdhQ200/200 mice. Scale bar $=50 \mathrm{~mm} . \mathrm{N}=4-5$ mice per condition, error bars represent S.E.M. and * $\mathrm{p}<0.05$.

A

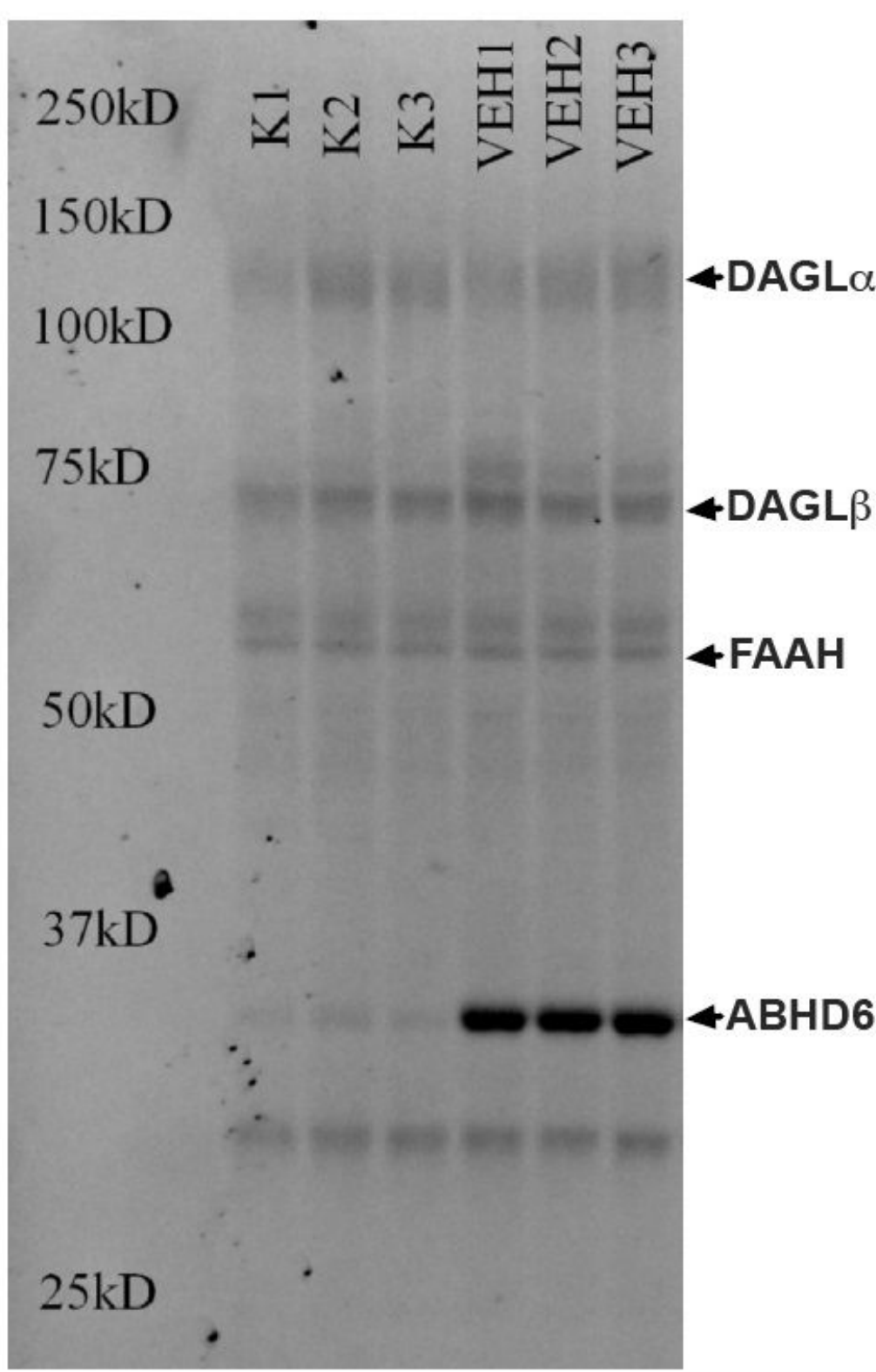

B
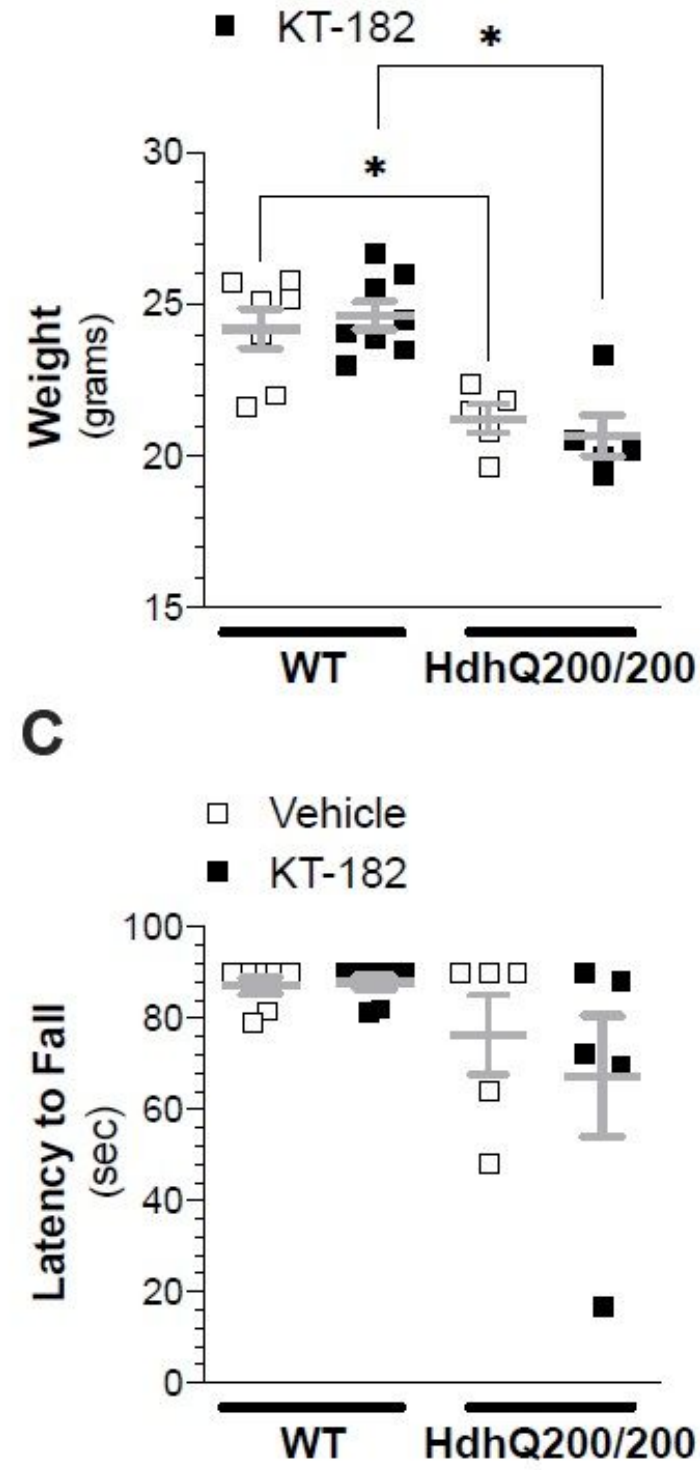

\section{Figure 3}

Semi-chronic, subcutaneous, osmotic pump delivery of KT-182 resulted in on-target inhibition of brain ABHD6 activity and did not affect overall body weight or grip strength of wild-type and HdhQ200/200 
mice at 10 months of age. Osmotic pumps delivering KT-182 were implanted subcutaneously (s.c.) in wild-type (WT) mice (12 weeks of age) for 10 days and ABHD 6 activity was measured by activity base protein profiling. A. 10 days s.c. osmotic delivery of KT-182 (lanes K1, K2 and K3) inhibited brain ABHD6 activity compared to vehicle-treated animals (lanes VEH1, VEH2 and VEH3) without affecting DAGLa, DAGLb and FAAH activity. $\mathrm{N}=3$ mice per treatment. B-C. Semi-chronic inhibition of ABHD6 did not affect (B) weight loss or (C) grip strength as measured by the inverted wire test of HdhQ200/200 mice compared to WT mice (10 months of age). Statistical analysis: One-way ANOVA analysis (multiple comparisons using Tukey) indicated significant reduction in overall body weight of HdhQ200/200 mice compared to WT and no significant difference in grip strength. $\mathrm{N}=5-8$ mice per condition, error bars represent S.E.M. and ${ }^{*} \mathrm{p}<0.05$. 


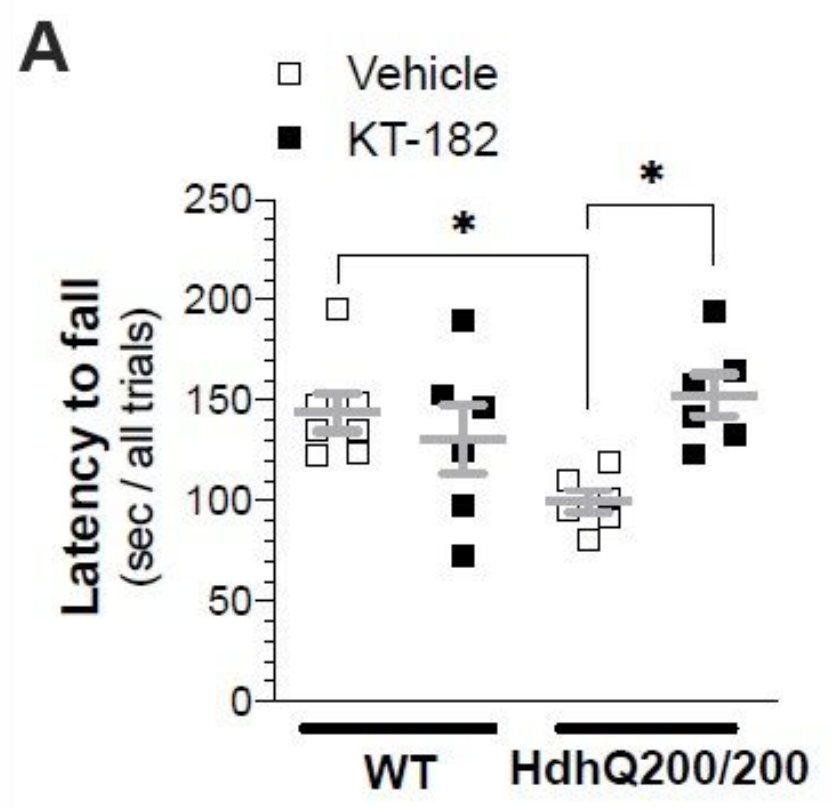

B

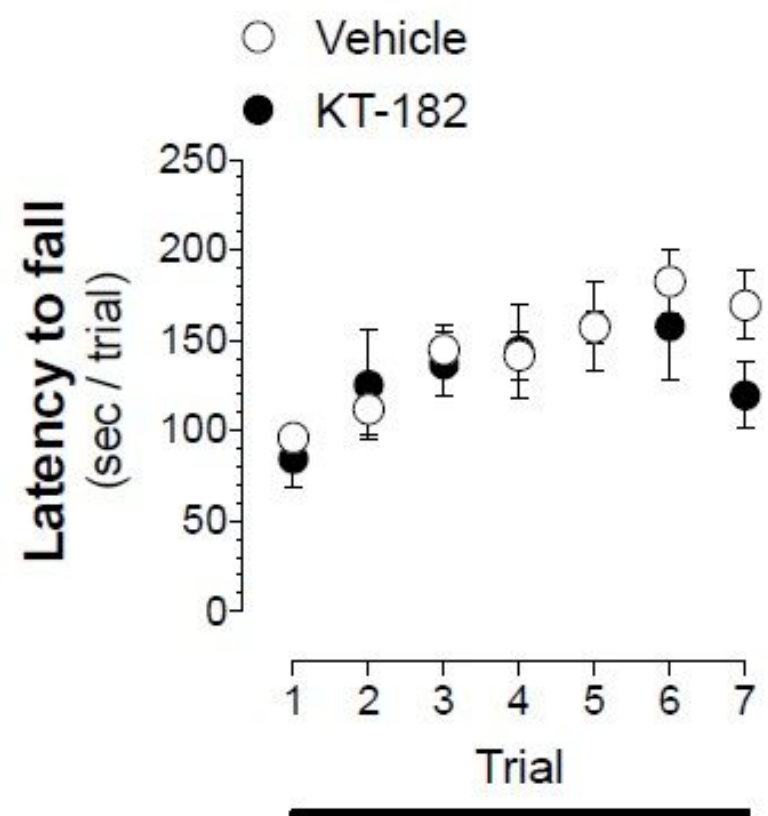

\section{WT}

C

D

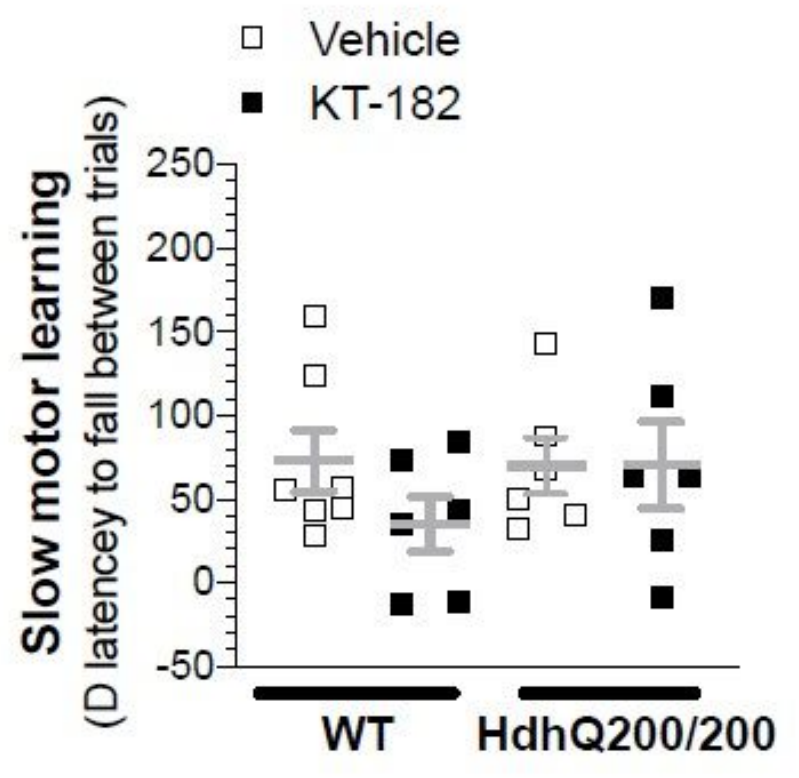

HdhQ200/200

Figure 4

Semi-chronic inhibition of ABHD6 rescued motor coordination deficits in HdhQ200/200 mice at 10 months of age. Motor coordination was measured in female wild type (WT) and HdhQ200/200 mice (10 months of age) that were implanted with osmotic pumps delivering either vehicle or KT-182. A. Analysis of average latency to fall from the rotarod measured across 7 trials showed that KT-182 did not affect motor coordination in WT mice but significantly rescued motor coordination in HdhQ200/200 mice. Statistical analysis: One-way ANOVA analysis (multiple comparisons using Tukey) indicated a 31\% 
reduction in latency to fall in HdhQ200/200 mice compared to WT mice and a $53 \%$ rescue of latency to fall in HdhQ200/200 mice when treated with KT-182. B. KT-182 treatment did not affect latency to fall in WT mice but C. enhanced latency to fall in HdhQ200/200 mice. Statistical analysis: Two-way ANOVA analysis with Bonferroni post-hoc analysis indicated significant rescue of motor coordination deficits in HdhQ200/200 by KT-182 compared to vehicle- treated HdhQ200/200 mice. D. Slow motor learning was comparable between HdhQ200/200 mice and WT littermates and not affected by treatment with KT-182. $N=6-7$ mice per condition, error bars represent S.E.M. and ${ }^{*} p<0.05,{ }^{*} p<0.01$.

A

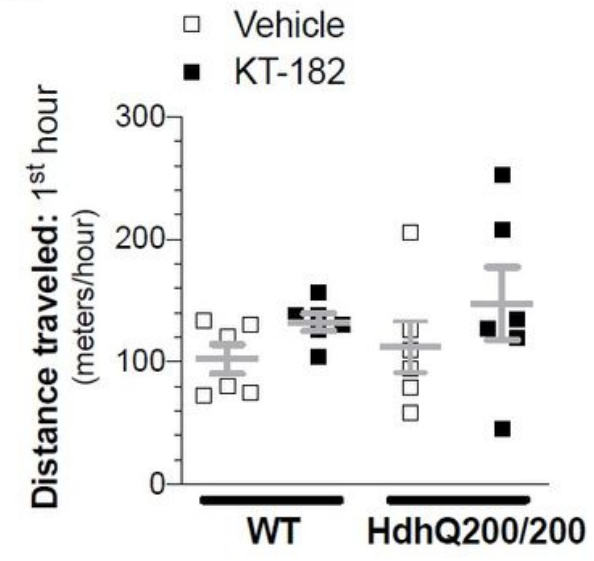

B

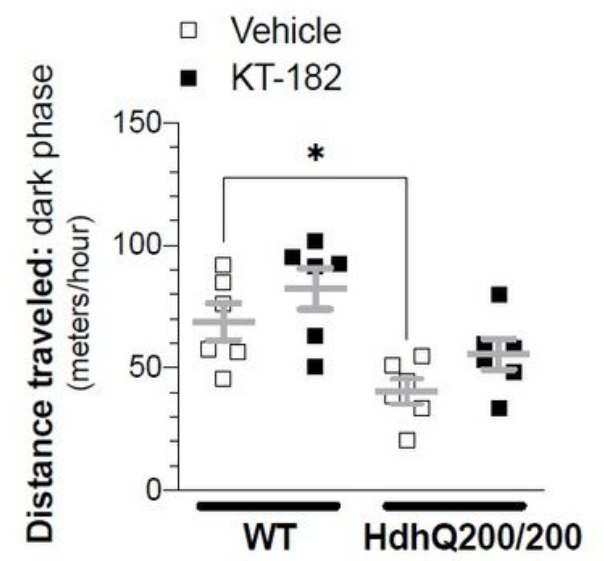

\section{C}

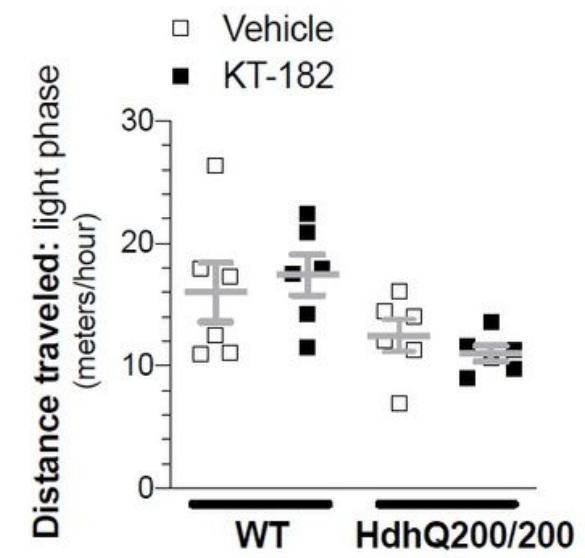

Figure 5

Semi-chronic inhibition of ABHD6 did not affect spontaneous locomotion of wild-type and HdhQ200/200 mice at 10 months of age. Spontaneous locomotion (meters/hour) was measured in female wild type (WT) and HdhQ200/200 mice (10 months of age) that were implanted with osmotic pumps delivering either vehicle or KT-182 using PhenoTyper chambers. A. Distance traveled during the first hour of entering the PhenoTyper chamber indicates exploration of a novel environment and was unaffected by genotype or KT-182 treatment. Distance traveled measured during the B. dark phase (high activity, 12 hours) and C. light phase (low activity, 12 hours). Statistical analysis: One-way ANOVA analysis (multiple comparisons using Tukey) indicated a 41\% reduction in spontaneous locomotion in HdhQ200/200 compared to WT measured during the dark phase but no difference between vehicle and KT-182. N = 6 mice per condition, error bars represent S.E.M. and * $p<0.05$.

\section{Supplementary Files}

This is a list of supplementary files associated with this preprint. Click to download.

- SupplementaryFigures.pdf 